\section{Crystal Structure of Isopropyllithium}

Ulrich Siemeling," Thomas Redecker, Beate Neumann, and Hans-Georg Stammler

Fakultät für Chemie der Universität Universitätstrasse 25, D-33615 Bielefeld, Germany

Received January 25, 1994

Organolithium reagents are of paramount importance in modern preparative chemistry, and our empirical knowledge concerning their chemical behavior is rather well-developed. ${ }^{1}$ Since their fascinating, and notoriously complex, structural chemistry can provide a key to a deeper understanding of the factors governing their reactivities, structural investigations are of great current interest. ${ }^{2}$ However, owing to the sensitivity of these compounds toward oxygen and moisture, X-ray structural investigations are often fraught with problems. This is especially true for the extremely reactive organolithium compounds $\mathrm{RLi}$ that are not stabilized by a Lewis base. There is thus a lack of information concerning the solid-state structures of these simple molecules.

To date, the only $\mathrm{X}$-ray structure determinations available for such "naked" organolithium compounds are those for $\mathrm{R}=\mathrm{Me},{ }^{3}$

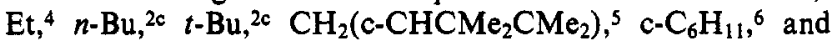
$\mathrm{CH}_{n}\left(\mathrm{SiMe}_{3}\right)_{3-n}\left(n=0,{ }^{7} 1,^{8} 2^{9}\right)$. [Bis(trimethylsilyl)methyl]lithium forms a polymeric chain structure; [tris(trimethylsily])methyl]lithium is dimeric in the solid state; methyl-, ethyl-, and tert-butyllithium crystallize as tetramers; [(trimethylsilyl)methyl]lithium, $n$-butyllithium, [(tetramethylcyclopropyl)methyl]lithium, and cyclohexyllithium are hexameric. Cyclohexyllithium represents a borderline case in this series of "naked" organolithium compounds since the crystals contain two molecules of benzene per hexamer; although benzene is not normally considered to be a Lewis basic solvent, there is evidence for weak donor interactions between benzene and organolithium compounds (vide infra).

We report the crystal structure of isopropyllithium, ${ }^{10}$ which is among the first examples of structurally characterized secondary alkyllithium reagents. ${ }^{11}$

Isopropyllithium is tetrameric in dilute $\left(<10^{-2} \mathrm{M}\right)$ cyclohexane solutions, but is predominantly hexameric in more concentrated solutions as demonstrated by cryoscopic measurements; benzene as the more Lewis basic solvent favors the existence of tetrameric units. ${ }^{12}$ The hexamer-tetramer equilibrium is shifted toward the hexamer upon lowering of the temperature as shown by NMR spectroscopic investigations of $2 \mathrm{M}$ solutions of $i$-PrLi in

(1) See, for example: (a) Schlosser, M. Struktur und Reaktivität polarer Organometalle; Springer-Verlag: Berlin, 1973. (b) Wakefield, B. J. The Chemistry of Organolithium Compounds; Pergamon Press: Oxford, 1974. (c) Wardell, J. L. In Comprehensive Organometallic Chemistry; Wilkinson, G., Stone, F. G. A., Abel, E. W., Eds.; Pergamon Press: Oxford, 1982; Vol. 1, Chapter 2. (d) Brandsma, L.; Verkruijsse, H. D. Preparative Polar Organometallic Chemistry; Springer-Verlag: Berlin, 1987; Vol. 1.

(2) Recent references: (a) Nichols, M. A.; Williard, P. G. J. Am. Chem Soc. 1993, 115, 1568. (b) Barnett, N. D. R.; Mulvey, R. E.; Clegg, W.; O'Neil, P. A. J. Am. Chem. Soc. 1993, 115, 1573. (c) Kottke, T.; Stalke, D. Angew. Chem. 1993, 105, 619; Angew. Chem., Int. Ed. Engl. 1993, 32, 580. Reviews: (d) Setzer, W. N.; Schleyer, P. v. R. Adv. Organomet. Chem. 1985 24, 353. (e) Weiss, E. Angew. Chem. 1993, 105, 1565; Angew. Chem., Int. Ed. Engl. 1993, 32, 1501.

(3) (a) Weiss, E.; Lucken, E. C. A. J. Organomet. Chem. 1964, 2, 197. (b) Weiss, E.; Hencken, G. J. Organomet. Chem. 1970, 21, 265.

(4) (a) Dietrich, H. Acta Crystallogr, 1963, 16, 681. (b) Dietrich, H. J. Organomet. Chem. 1981, 205, 291.

(5) Maercker, A.; Bsata, M.; Buchmeier, W.; Engelen, B. Chem. Ber. 1984, $117,2547$.

(6) Zerger, R.; Rhine, W.; Stucky, G. J. Am. Chem. Soc. 1974, 96, 6048.

(7) Hiller, W.; Layh, M.; Uhl, W. Angew. Chem. 1991, 103, 339; Angew. Chem., Int. Ed. Engl. 1991, 30, 324.

(8) Atwood, J. L.; Fjeldberg, T.; Lappert, M. F.; Luong-Thi, R.; Shakir, N. T.; Thorne, A. J. J. Chem. Soc., Chem. Commun. 1984, 1163.

(9) Tecle', B.; Magsudur Rahman, A. F. M.; Oliver, J. P. J. Organomet. Chem. 1986, 317, 267.

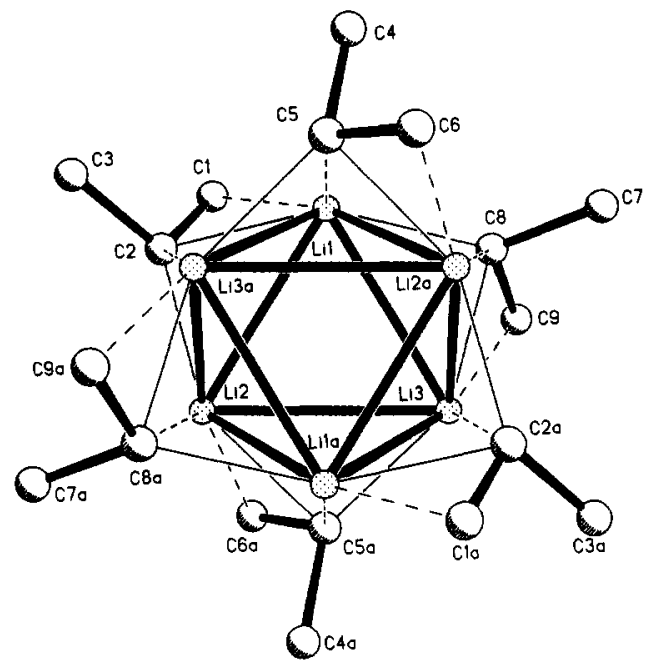

Figure 1. Molecular structure of $(i-\mathrm{PrLi})_{6}$. Hydrogen atoms are omitted for clarity. Solid lines signify short and dashed lines signify long Li-C distances. Selected bond lengths $(\AA)$ and angles (deg): $\mathrm{C} 1-\mathrm{C} 21.531$ (2), C2-C3 1.533(2), C4-C5 1.528(2), C5-C6 1.541(2), C7-C8 1.533(2), C8-C9 1.541(2); C1-C2-C3 109.2(2), C4-C5-C6 107.99(13), C7C8-C9 108.00(13).

cyclopentane. ${ }^{13}$ When a $1.86 \mathrm{M}$ solution of isopropyllithium in $n$-hexane ${ }^{14}$ is allowed to stand at $-35^{\circ} \mathrm{C}$ for several days, the hexamer is obtained as colorless platelets.

In contrast to methyl-and ethyllithium there are no interactions between the oligomeric units in the crystal (the shortest $\mathrm{Li}-\mathrm{C}$ distance between two aggregates is $4.13 \AA$ for $i$-PrLi and 2.52 $\AA$ for both $\mathrm{MeLi}$ and $\mathrm{EtLi}$ ).

The crystal structure of isopropyllithium resembles those of $n$-butyllithium ${ }^{2 \mathrm{c}}$ and benzene-solvated cyclohexyllithium. ${ }^{6}$ The six lithium atoms form a distorted octahedron with six short [2.388(3)-2.404(3), average $2.395 \AA$ ] and six long [2.926(3)$3.014(3)$, average $2.959 \AA] \mathrm{Li}-\mathrm{Li}$ distances (Figure 1).

Six of the eight faces of the octahedron are each capped by a single $i$-Pr unit. Two almost equilateral (and opposite) triangular faces remain uncapped; it is noteworthy that in $\left(\mathrm{C}_{6} \mathrm{H}_{11} \mathrm{Li}\right)_{6} \cdot 2 \mathrm{C}_{6} \mathrm{H}_{6}$ the two benzene molecules are positioned parallel above the two uncapped faces. The secondary carbon atom $\left(\mathrm{C}_{\alpha}\right)$ of an $i$-Pr unit coordinates the two lithium atoms that form the longest side of a capped triangular face [distances: $2.160(3)-2.197(3)$, average $2.180 \AA]$. The distance between $\mathrm{C}_{\alpha}$ and the third lithium atom of a capped triangular face is significantly longer [2.297(3)$2.316(3)$, average $2.308 \AA]$; at an almost identical distance from this lithium atom is a methyl carbon atom $\left(C_{\beta}\right)$ of a neighboring

(10) Crystallographic data: data collection on a Syntex $P 2_{1}$ four-circle diffractometer with graphite-monochromated Mo $\mathrm{K} \alpha$ radiation; structure solution with direct methods, full-matrix least-squares refinement on $F^{2}$ (Siemens SHELXTL plus, SHELXL-93). $R$ values: $R_{1}=\Sigma|| F_{0}-\left|F_{\mathrm{c}}\right| / \Sigma\left|F_{\mathrm{o}}\right|$ $w R_{2}=\left[\sum w\left(F_{0}^{2}-F_{c}^{2}\right)^{2} / \sum w F_{0}^{2}\right]^{1 / 2} . C_{3} \mathrm{H}_{7} \mathrm{Li}$, space group $P 1, Z=12, a=$ $9.272(3) \AA, b=10.049(3) \AA, c=13.734(4) \AA, \alpha=85.12(2)^{\circ}, \beta=84.77(2)^{\circ}$, $\gamma=63.75(2)^{\circ}, V=1141.5(6) \AA^{3}, d_{\text {calcd }}=0.873 \mathrm{Mg} \mathrm{m}^{-3} ; 6984$ reflections, 6617 unique observed, were measured at $173 \mathrm{~K}$. The non-hydrogen atoms were refined anisotropically; all hydrogen atoms were located by difference Fourier synthesis and refined free isotropically. $R_{1}=0.0584$ calculated from 4039 reflections with $F_{0} \geq 4 \sigma\left(F_{0}\right) ; w R_{2}=0.1394$ for 385 parameters (no restraints). The asymmetric unit contains two crystallographically independent hexamers (each on a center of inversion); they are identical within the margin of error of the structure determination.

(11) Benzene-solvated cyclohexyllithium $\left(\mathrm{C}_{6} \mathrm{H}_{11} \mathrm{Li}\right)_{6} \cdot 2 \mathrm{C}_{6} \mathrm{H}_{6}$ is the only secondary alkyllithium compound for which crystallographic data are available (ref 6). The structure of cyclopentyllithium has been reported to be isotypic with that of cyclohexyllithium (ref 2c); however, crystallographic data have not been published so far.

(12) Lewis, H. L.; Brown, T. L. J. Am. Chem. Soc. 1970, 92, 4664

(13) Thomas, R. D.; Jensen, R. M.; Young, T. C. Organometallics 1987 , 6,565 .

(14) Prepared under argon from lithium sand and isopropyl chloride in $n$-hexane according to a published procedure (Gilman, H.; Moore, F. W.; Baine, O. J. Am. Chem. Soc. 1941, 63, 2479). 


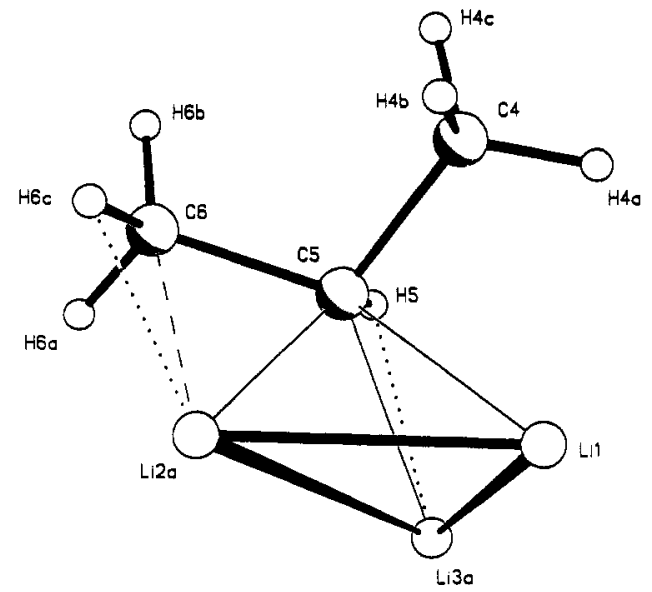

Figure 2. View of one of the Li triangles of the distorted octahedron with a coordinated $i$-Pr group. Selected bond lengths $(\AA)$ and angles (deg): C4-H4a 1.04(2), C5-H5 0.99(2), C6-H6a 0.97(2), C6-H6b 1.00(2), C6-H6c 1.04(2), C4-H4b 1.05(2), C4-H4c0.99(2); C5-C4-H4a 113.2(11), C5-C4-H4b 111.4(11), C5-C4-H4c 116.5(10), C4-C5-H5 103.8. (9), C6-C5-H5 103.0(9), C5-C6-H6a 114.8(13), C5-C6-H6b 112.9(10), C5-C6-H6c 111.8(10).

$i$-Pr unit $[2.302(3)-2.314(3)$, average $2.308 \AA]$. Thus, each lithium atom is in contact with four carbon and four lithium atoms, respectively, and two short and two long distances are observed in each case. Additionally, the conformation has rather short $\mathrm{Li}-\mathrm{H}$ distances (Figure 2), with the $\mathrm{Li}-\mathrm{H}_{\alpha}$ distances (1.9081.965 , average $1.936 \AA$ ) being significantly shorter than the $\mathrm{Li}-$ $\mathrm{H}_{\beta}$ distances (2.005-2.089, average $2.038 \AA$ ); strong $\mathrm{Li}-\mathrm{H}_{\beta}$ interactions are discussed to be responsible for the thermal decomposition of alkyllithium species via a $\beta$ elimination pathway. ${ }^{15}$

Our structure determination of isopropyllithium closes a gap between the structurally characterized primary and tertiary alkyllithium reagents. As for $n-\mathrm{BuLi}_{,}{ }^{16} t-\mathrm{BuLi}^{17}$ and (c-CHCMe $2_{2}^{-}$

(15) Houk, K. N.; Roudan, N. G.; Schleyer, P. v. R.; Kaufman, E.; Clark, T. J. Am. Chem. Soc. 1985, 107, 2821 and references therein.
$\left.\mathrm{CMe}_{2}\right) \mathrm{CH}_{2} \mathrm{Li},{ }^{18} \mathrm{Li}-\mathrm{C}_{\beta}$ interactions are an important structural feature for $i-\mathrm{PrLi}$. Obviously, it is more favorable for all four compounds that a lithium atom interacts with four carbon atoms via two short and two long distances, rather than with only three carbon atoms via three short distances, which would correspond to a perfectly symmetric capping of a triangular face. The $\mathrm{Li}-\mathrm{C}_{\beta}$ interactions within an oligomeric unit allow the existence of isolated aggregates in the crystal. For hexameric $\mathrm{Me}_{3} \mathrm{SiCH}_{2} \mathrm{Li}$ and dimeric $\left(\mathrm{Me}_{3} \mathrm{Si}\right)_{3} \mathrm{CLi}$, which do not contain $\mathrm{C}_{\beta}$ atoms, $\mathrm{Li}-\mathrm{C}_{\gamma}$ interactions are observed instead. ${ }^{19}$ In the case of $\left(\mathrm{Me}_{3} \mathrm{Si}\right)_{2} \mathrm{CHLi}$, $\mathrm{EtLi}$, and $\mathrm{MeLi}$, respectively, the subunits are associated through $\mathrm{Li}-\mathrm{C}_{\alpha}$ interactions and form a one-, two-, and three-dimensional array, respectively.

It is remarkable that the basic structure of $\left(\mathrm{C}_{6} \mathrm{H}_{11} \mathrm{Li}\right)_{6} \cdot 2 \mathrm{C}_{6} \mathrm{H}_{6}$ is quite similar to that of $(i-\mathrm{PrLi})_{6}$. Obviously, the influence of the benzene molecules on the hexameric unit of cyclohexyllithium is almost negligible; this is in contrast to the well-documented depolymerizing effect of benzene on the aggregation of alkyllithium compounds with a moderately bulky $\mathbf{R}$ group in solution. ${ }^{\text {la, } 12}$

Acknowledgment. This work was financed by the Deutsche Forschungsgemeinschaft. U.S. thanks professor Dr. P. Jutzi for his continued support.

Supplementary Material Available: Details of the X-ray analysis and tables of atomic coordinates, bond lengths and angles, and thermal parameters (14 pages); listing of observed and calculated structure factors ( 15 pages). This material is contained in many libraries on microfiche, immediately follows this article in the microfilm version of the journal, and can be ordered from the ACS; see any current masthead page for ordering information.

(16) The average $\mathrm{Li}-\mathrm{C}_{\beta}$ distance is $2.29 \AA$ (ref $2 \mathrm{c}$ ).

(17) The average $\mathrm{Li}-\mathrm{C}_{\beta}$ distance is $2.37 \AA$ (ref $2 \mathrm{c}$ ).

(18) The average $\mathrm{Li}-\mathrm{C}_{\beta}$ distance is $2.37 \mathrm{~A}$ (calculated from the crystallographic data given in ref 5 ).

(19) The relevant $\mathrm{Li}-\mathrm{C}_{\gamma}$ distances range from 2.47 to $2.54 \mathrm{~A}$ for ( $\mathrm{Me}_{3}$ $\mathrm{Si})_{3} \mathrm{CLi}$ (ref 7) and from 2.59 to $2.85 \AA$ for $\mathrm{Me}_{3} \mathrm{SiCH}_{2} \mathrm{Li}$ (calculated from the crystallographic data given in ref 9); these values are similar to the interaggregate $\mathrm{Li}-\mathrm{C}$ distances in MeLi (refs 3) and EtLi (refs 4). 